\title{
Design of an Adaptive Near Field Communication Technology using Finite State Machine within Web Services
}

\author{
Khalid Aldriwish
}

\begin{abstract}
The ongoing Web success can be related to its scalability and sustainability. Web Services (WSs) can provide different patterns for delivering software application abilities and the models by which enterprises and associated parties will trade. WSs represent a flexible way to offer existing components available to applications through the Internet. WSs are a new trend that shares ubiquitous systems with others. The popularity of Web is increasing with their associated systems. These systems play a significant key role as their compromise has a technical effect, and their need is rising. One of these systems is called Technology wireless (NFC). NFC is becoming an active area nowadays. The complexity of setting the NFC increases as the application, system architecture, and associated parts must satisfy various requirements of ever involving project scenarios. Several organizations are getting the benefit from the NFC. This paper will explore and develop an approach of developing a technique that will permit to exchange by technology wireless (NFC) data between the parties within a Web services environment. This technique has an advantage that facilitates the possibility of adopting such a system to decrease the time-cost and effort for staff and customers. Several means of mobile payments technique have been entitled and adopted this service in many countries, e.g., Europe, USA, Asia, and in the Middle East such as Saudi Arabia. Many users of these countries using a smartphone to pay services via their mobile phones for a wide range of activities. Consequently, this paper involves an agile technique of validating Finite State Machine (FSM) and JFLAP tools.
\end{abstract}

Keywords: Web Service, NFC and Mobile payment system (MPS), FSM and JFlap

\section{INTRODUCTION}

$\mathrm{T}$ he ongoing world is moving towards reducing using a cash money society and concentrating on real mobility and commercial service evolution years onward. Also, wireless technology (Near Field Communication) is a technique that allows exchanging data in the range of $10 \mathrm{~cm}$ between parties, and that globally operates at frequency $13.56 \mathrm{MHz}$, so the transfers data at $474 \mathrm{~kb}$ per second. NFC is a collection of criteria for smartphones and modern devices, e.g., phones, iPad, to launch radio communication with each other by moving them close together. NFC technology can help to

Manuscript received on August 20, 2021.

Revised Manuscript received on August 24, 2021.

Manuscript published on August 30, 2021.

* Correspondence Author

Khalid Aldriwish*, Computer Science department, College of Science and Humanities of Al-Ghat, Majmaah University, Majmaah, Saudi Arabia. Email: k.aldriwish@mu.edu.sa

(c) The Authors. Published by Blue Eyes Intelligence Engineering and Sciences Publication (BEIESP). This is an open access article under the CC BY-NC-ND license (http://creativecommons.org/licenses/by-nc-nd/4.0/) perform purchases through smartphones. Moreover, NFC is a short-range wireless RFID technology that builds interacting electromagnetic radio fields instead of the traditional direct radio transmissions operated by technologies such as Bluetooth. However, mobile payment techniques firstly appeared in some great countries, e.g., Japan, and then spread to several countries, such as the UK, USA, UAE, and recently Saudi Arabia and Qatar [1].

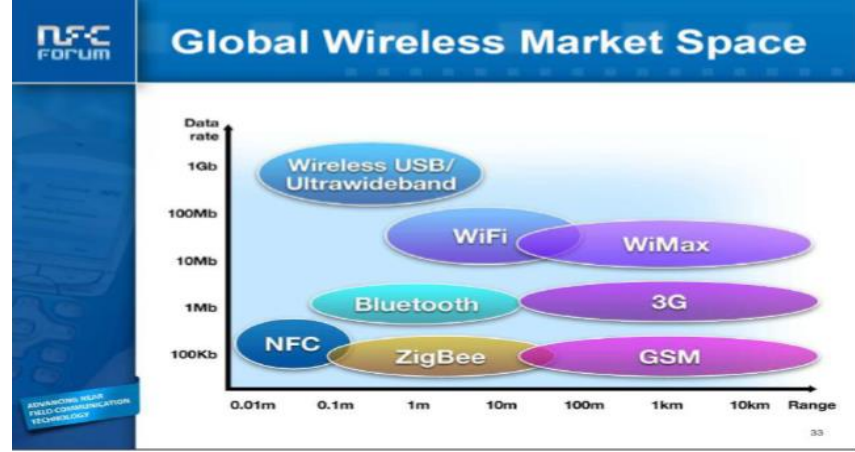

Fig. 1. The universal wireless connections in meters and their connection speeds in bites.

Firstly, the great companies in the world have launched a promotion of using smartphones performing services. Some of these companies are Google, Samsung, Apple, and Nokia, which have adopted this technology. Yet, NFC is described as a method that authorities two dynamic smart devices with chips convey small pieces of data between each other when they are nearby $1 \mathrm{CM}-4 \mathrm{CM}$ via short-range wireless connection and at low speeds of 106-414 kbps, based on the system configurations [10].

The NFC Forum is established by some largest companies, such Sony, NXP Semiconductors, and Nokia. NFC has known and becomes a standard model for the International Organization for Standardization (ISO). It is a set of a consortium that represents criteria on top of the ISO standards to confirm maximum compatibility and capability across all implementations of NFC technology [10].

In 2003, Coca-Cola Company in Japan has used and tested the purchase of a soft drink from a vending machine through a phone; this technology has been available in the country for a long time, allowing consumers to pay for a purchase by simply waving their device over a reader the point of sale $[2,5]$.

Published By: Blue Eyes Intelligence Engineering and Sciences Publication

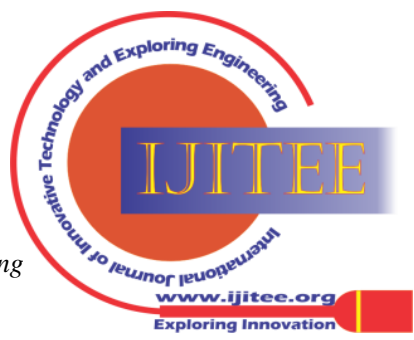


Moreover, in April 2013 - MasterCard in Canada was declared and launch MasterPass. This project shows and provides the upcoming facility of digital service payments, which becoming Canadian consumers are the first persons in North America can be registered their MasterPass services. The MasterPass is an innovation that delivers and generates value for consumers and merchants through its simplicity and security. It has many advantages, e.g., facilitating a seamless, trustful, secure, and speedy check out of where consumers may be and what screen they may be using. It saves the information of credit cards and shipping information in a reliable cloud hosted by the trusted entity and has high integrity. [3].

Furthermore, many purchase methods are used by others, e.g., cash, debit, or credit cards, and now by mobile phone method. The mobile web payment technique can be used to pay via permitting consumers to enter their information when making the payment. For instance, if a vendor makes a payment method automatically and securely recognizes customers, card details can be recalled and restored for upcoming purchases. Turning credit card payments into simple single click-to-buy highly provides conversion rates for further purchases [2].

Nevertheless, Web Services are discovered to provide interoperability between diverse applications and their associated parts. The main advantage of Web services is the interaction between different platforms and language-independent interfaces. Web Services allowed the integrating of various systems regardless of their platforms. The universal description, discovery, and integration (UDDI), Web Services description language (WSDL), and simple object access protocol (SOAP) describe standards for service discovery, description, and messaging protocols. Web services are standardized methods to propagate communication between the client and server applications via the WWW (World Wide Web) [7,8].

\section{STATEMENT AND CONTRIBUTIONS}

NFC works by utilizing electromagnetic radio fields with their associated parties while recent technologies, viz, Bluetooth and Wi-Fi, concentrate and enable radio transmissions.

This paper will primarily show how to ensure that the NFC technology is feasible and workable based on the Web services environment, which should decrease time-cost and effort for staff and customers. As a result, the contributions will be as the following:

- Set up and propose an approach that uses a mobile payment system (MPS) that works as NFC technology which should ease and facilitate customer's services in different places, e.g., car parking, restaurants, shopping malls, petrol stations...etc.

- Customize and explore the design of the MPS by leaving essential degrees of freedom relating to their structure, by granting some features in dynamically changing environments.

- Tame and address most issues by examining MPS to prove and validate the proposed approach to obtain an optimal solution using FSM and JFLAP tools.

- Implement and design a prototype structure of NFC technology to manage the system specifications and requirements, utilizing a systematic method that can authorize based on the model standards.

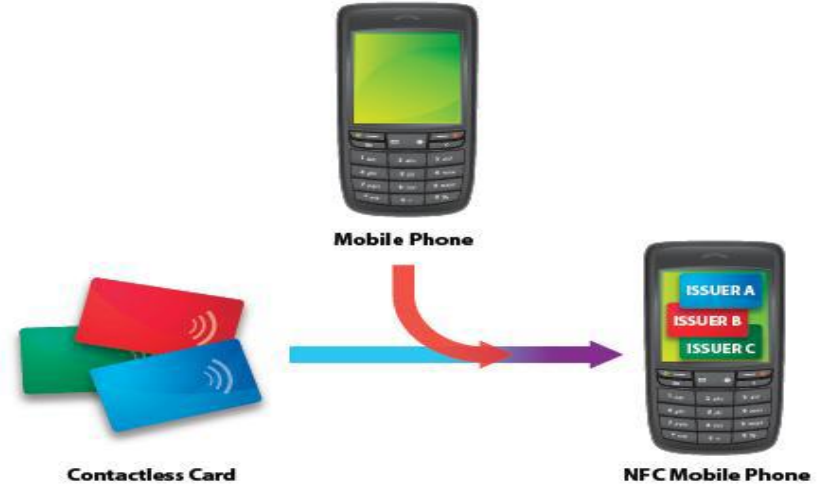

Fig. 2. Concept of an NFC phone.

As shown in Fig 2, NFC provides and offers consumers paying their utility services and goods through their mobile smartphones by touching or passing them over another NFC-equipped device viz. a register or terminal. And the funds should be transferred from the customer's debit/credit card account stored through the mobile smartphones via the phone's wallet, e.g., iPhones, Samsung. [10]

In addition, the phone SIM card is compatible with NFC, so your mobile phone will functionally work as a credit card. Through NFC technology, MPSs have become more concerned than a traditional credit cards. It is an attractive market for new entrants as it can be used a credit card, smartphones, and wristbands, where enabling them to pay for their use such as toll roads, car parking, restaurants, and petrol stations.

The paper is organized as the following: Section I produce an introduction of NFC and Web services. The problematics and contributions are presented in section 2. Section 3 demonstrates the Proposed Architecture of MPS. The Discussion is provided in Section 4. And implementation is described in section 5. Section 6 presented the critical evaluation and validation of the MPS. An algorithm of MPS approach shown in section 7. Finally, there is the concluding remark.

\section{PROPOSED ARCHITECTURE}

NFC is a set of forms of contactless communication between smart devices, e.g., smartphones, touch, tablets. Contactless communication lets a client wave and passes the smartphone over an NFC-compatible device to send information without requiring to touch the devices together by hands.

More, a proposed architecture of MPS has been used to manage and raise the connectivity and simplicity of NFC technology, to increase the efficiency and facilitating the processing of purchase transactions for customers. Fig 3 illustrates the proposed architecture diagram of the MPS, which enables some elements that are employed to manage the streaming of data flow of the system. The feasibility of MPS can be reached in most modern technology based on wireless technology.

\section{Published By:}

Blue Eyes Intelligence Engineering and Sciences Publication

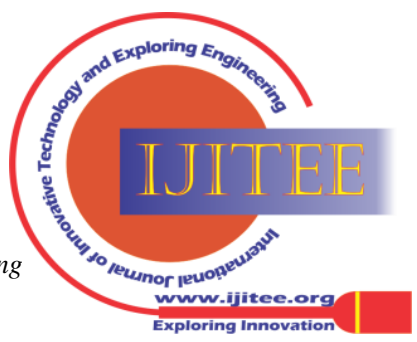




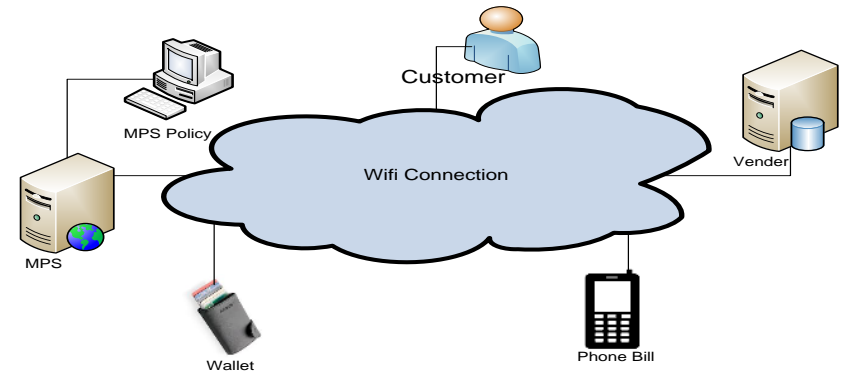

Fig. 3. Shows a proposed architecture of MPS

The WIFI network increases the connectivity and the outcomes. This proposed architecture shows the sources and processes that take place within the system and outputs. Sources in this system are the vendor and customers, allowing them to access the MPS software and conduct operations available to them. Nonetheless, the proposed architecture includes some entities, e.g., MPS, vendor, customer, wallet, phone bill, and MPS policy, to control data streaming.

\section{DISCUSSION}

The use case diagram has explained MPS processes, and several organizations have used case diagrams to create such a system to facilitate the feasibility and adaptability of the system. The main advantage here is that it hints at defining the approach scope. Moreover, the model is designed using Unified Modeling Language (UML). The UML allows visualization of the system's design dynamically through diagrams.[10]

The use case of the MPS plays an important role in showing the requirement technique that has widely demonstrated a key characteristic of the process of the system. However, the MPS use case is a list of stages that defines interactions between a vital role within the system to achieve a goal. As presented in Fig 4, the use case states the specification of sequences of actions within the system, subsystem, and class that can perform by interacting with outside actors.

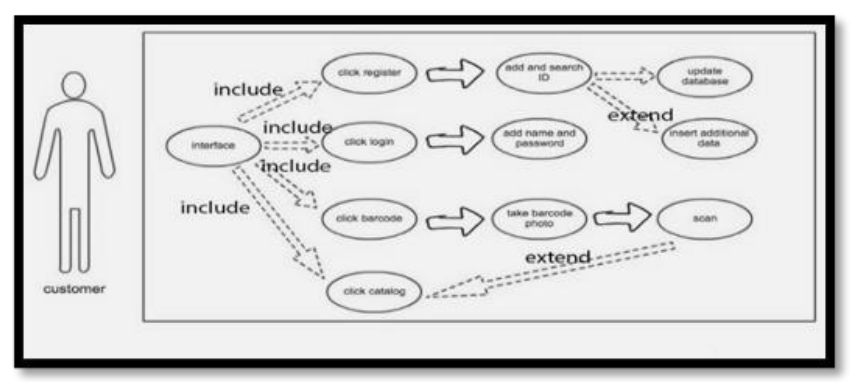

Fig. 4. Use case of building MPS

We have one actor called a customer in this use case, and an interface will be the main menu. This use case includes four other use cases: register, login, barcode scan, and catalog. In addition, when the new customer chooses to enter the interface and click register, it will add the customer's ID and then search about this ID in the program. Next to the data in the database will be updated automatically. Still, when the customer has completed the registration, the customer will click the login to add a name and password. The third condition is that when the customer clicks the barcode button and takes a photo of the product willing to buy, the program will scan, and the customer will transform from barcode to click catalog to choose the product.

\section{DATA DICTIONARY}

Firstly, analyzing a system of objects with which users should interact to classify each object and its association to other objects. This method means that data modeling and leads result in a picture of object association parts. When each data object is given a descriptive name, its relationship is described, the type of data viz. text or image or binary value is identified. Predefined values are recorded, and a short textual explanation is shown. This group can be organized for reference into a book called a data dictionary [9]. Data Dictionary, called data bank repository, collects names, definitions, attributes, and elements about data descriptions used or captured in a database, information system[6].

Once designing and making a software program that uses the data model, the bank of data dictionary should be consulted. To realize where a data item should fit in the structure, what values it may have, and basically what the data item means in real-world terms. [9].

\section{SYSTEM IMPLEMENTATION}

Java Database Connectivity (JDBC) shows a database connection and is easy to use with the MySQL database engine. We have designed and implemented the SR barcode program by using computer object-oriented programming Sun Java which acting as NFC technology, as developers can make and use it on the client and server sides, permitting it to execute as a distributed system anywhere. The database is used to store dataset during any transactions. Moreover, this database is an important key and should be created based on the system requirements. These requirements drive state and define a set of database tables and the types of relationships among them.

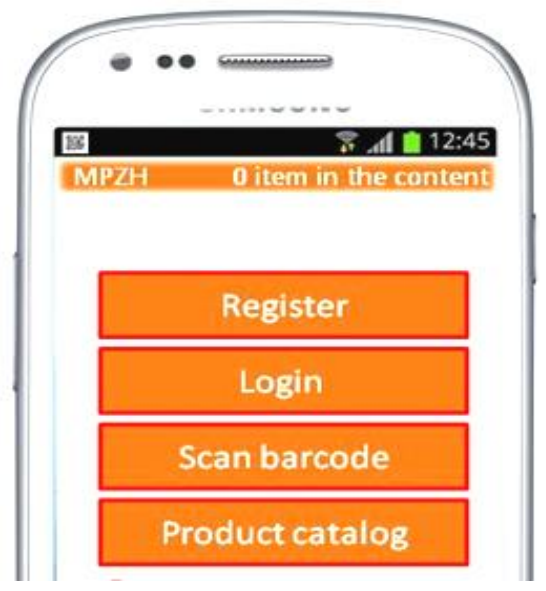

Fig. 5. Shows program interface

SR barcode program works on a smartphone to handle and facilitate purchasing from any store viz. malls, restaurants, petrol stations...etc., in their usage. The SR barcode can be working with barcode scanners, payment processing systems, and mobile smartphones.

Published By:

Blue Eyes Intelligence Engineering and Sciences Publication

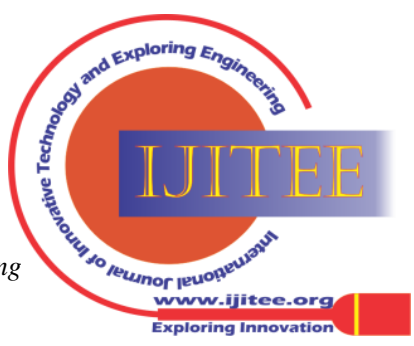


It can be easy to read information and " pick up" special offers, coupons, and discounts from smart posters or smart billboards. Users or customers with a smartphone have the ability to use a camera and suitable scanning program to scan the image of SR code which activates the data stored that provides a speedy and effective way for customers to receive information about the product in a fully new way.

However, most vCards have primary contact information fields, i.e., phone numbers, address information, Email address and the Internet URLs, but they are not limited to those. Over the years, the standard has been revised to include new fields and data types. vCards involve vital directory information names, and most vCards have features, e.g., graphics and multimedia, photographs, company logos, and audio clips such as for name pronunciation. Geographic and time zone information in vCards lets others know when to contact you. Furthermore, vCards support multiple languages, i.e., English. The vCard specification is transport and operating system independent so that you can have vCard-ready software on any computer [4]

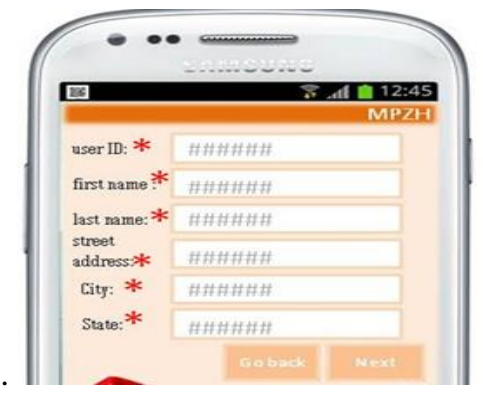

Fig. 6. shows the program system adding information

As stated in Fig 6,7,8 and 9 respectively, any new user or customer must register with this program for usage. It requires adding an ID user, name, address, city...etc., and every user must enter their information to access the program system. However, the way to access the program system is user_id and password, so, every figures indicated and showed its job.

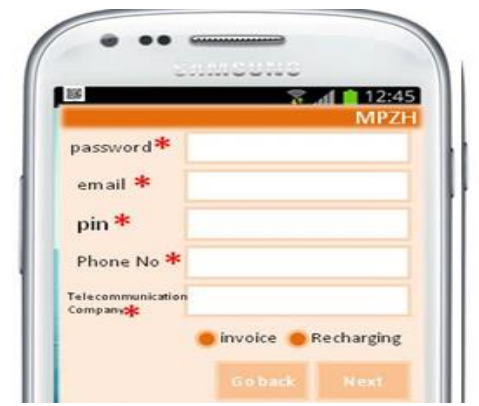

Fig. 7. shows how to access the program system

Still, the program system requires to active payment system via smartphone as the wallet app in iPhone so, an algorithm will encrypt all information on debit/credit cards.

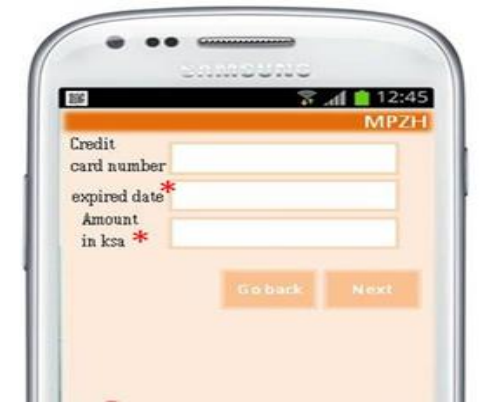

Fig. 8. shows how to enter cards information

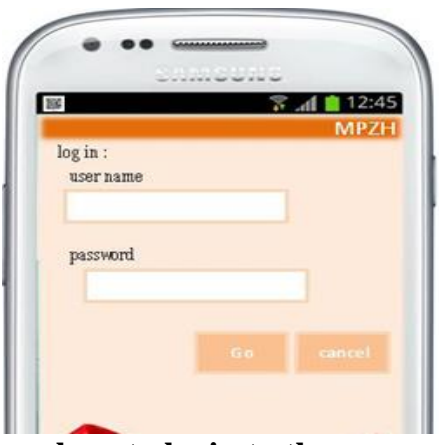

Fig. 9. shows how to login to the program system

\section{Evaluation and validation of the MPS by FSM and JFLAP} tool

A proposal technique of the MPS has tackled and tamed most issues and gives a suitable result that is reusable in similar contexts. The major advantage of this technique is to prove the feasibility of the proposed approach over the Web environment by exploring them via FSM and JFLAP tools and then results will provide the ability to accomplish eligible adaptability to be able to progress system performance of the MPS in different environments via using the existing resources and activities.

Multi-Agent System (MAS)

An agent is a vigorous tool in modern technology. It is a software application program that interconnects with other software programs, joining and responding to behavior, acting, and linking with existing assets on demand. Every single agent has a collection of attributes, i.e., size, capability, and speed. Also, MAS is the sub eld of artificial intelligence that aims to represent principles for designing composite and heterogeneous systems concerning several interacting agents; also, MAS has more interest in research pace because of the high advantages found within such systems. For instance, they can deal with huge issues, for a single-agent system is quicker and more dependable. They can solve problematic focus on communication, coordination, and negotiation [11].
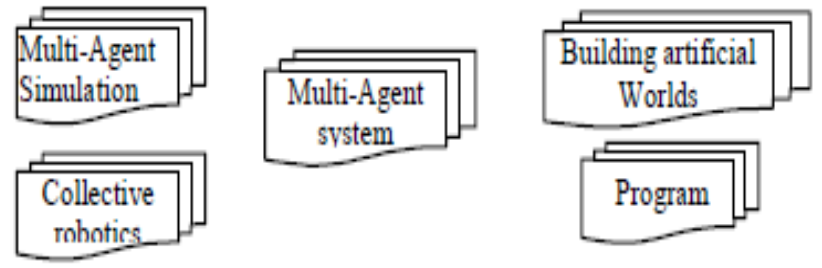

Fig. 10. shows application domains in MAS

\section{Finite State Machine (FSM)}

FSM, occasionally called state chart, has been used and extended in a wider range of applications and should be found in all pieces of progress in sequential digital hardware. It is used and worked by moving from one state to another and releases output figures or symbols in response to the input symbols as received from the environment. It is a pattern that describes the existing required behavior of implementation; it includes a data structure that is used to show and provide activities and actions with a sequence of incidents [11].

Published By:

Blue Eyes Intelligence Engineering

and Sciences Publication

DOI: 10.35940/ijitee.J9448.08101021

Journal Website: $\underline{w w w . i j i t e e . o r g}$

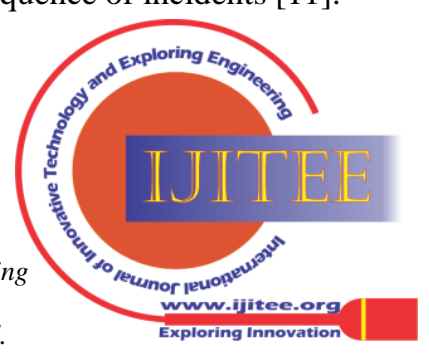


This part will explore and verify the feasibility of MPS working with FSM and how this technique will support the model to be validated and executed. This stage will explain how to define a finite state machine using JFLAP and then showing coding by SUN Java.

\section{JFLAP}

JFLAP is a set of graphical tools that can be used to improve the fundamental concepts of formal Languages and automata theory. It is a collection of instructional software that uses for showing high accuracy of experiments with grammar automata. The most feature of JFLAP is the ability to experimentation with applications and proofs. Also, JFLAP can experiment with grammars and theoretical machines [11].

\section{JFLAP WITH Design FSM}

This part will investigate the design of an FSM with five states in the model: the first state is the beginning of the search of states/objects called Search in the system's database; more than one can be searched at the same time. Next, all the services have to register with SReg to be included and allocated. Also, every single customer can find out a store to pay their bills which needs. Then, every single vendor has to register with the system to ensure they can benefit via online services in their stores. However, any vendor that does not profits regarding MPS policy.

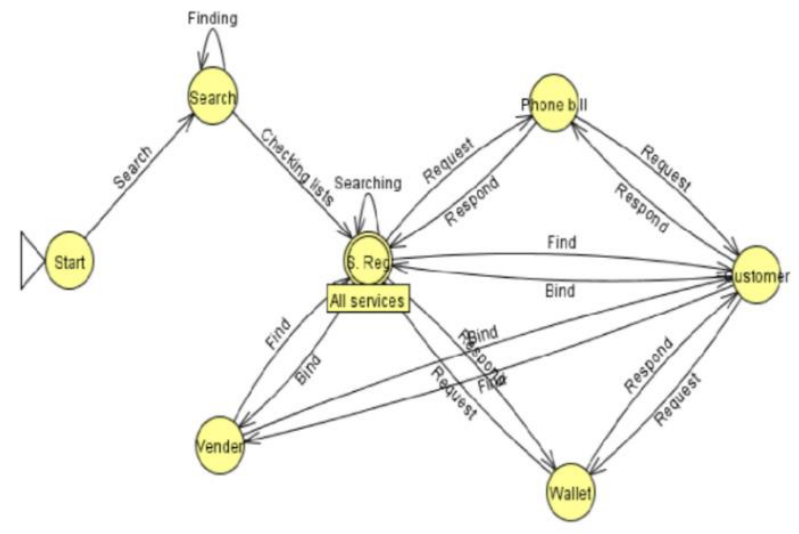

Fig. 11. Shows the MPS diagram designed by an FSM and JFLAP to verify and validate the proposed approach.

As stated in fig 11, the main key role of using FSM is to make the visibility pure so, facilitating the proposed approach of the MPS by entailing with the progress to reach proper solutions. The FSM is a composite of applications into simple classified search steps in the overall model. It supports and provides developers and designers a good feasible visualization of the model and defines each stage of the approach. Hence, we review these states by testing high probabilities to accomplish required outcomes via the test techniques to obtain the best possible results. Consequently, these tests/assessments should declare the validation and possibility of the practical applicability of the model by stating a green sign with each state with standard results.

Also, in fig 11, the model scenario shows that all these components interact and chat with each other based on MPS's policy requirements. Hence, the customer, vendor, phone bill, wallet are black-box services that cooperate with each other for increasing progress and connectivity. reach and allocated in the system should not receive any

\section{Test Step by State}

As we mentioned above, there are two tests; first, the Step by State facilitates and supports to execute of the model by using state by state in moving to another state: if green color, it will eventually be a final state of green color, if there are any problems between each state, it will be providing and shown by red color.

\section{THE ALGORITHM FOR THE MPS APPROACH}

Pseudo code is a set of processes that demonstrates a collection of high-level description of a computer programming algorithm. As declared in algorithm method of MPS(), it shows and describes how services can be registers as venders/customers which have inserted in the model based of MPS's policy. This algorithm has proved the compact of examining the main method of the proposed technique which is impacted on the MPS system.

Additionally, this algorithm has typically omitted the details that are not important for understanding the algorithm, e.g., variable declarations. The advantage of using pseudo code is that having and giving the ability to understand it more easily than traditional programming language code. More, It is sketching out the structure of the programme before the actual coding takes place.

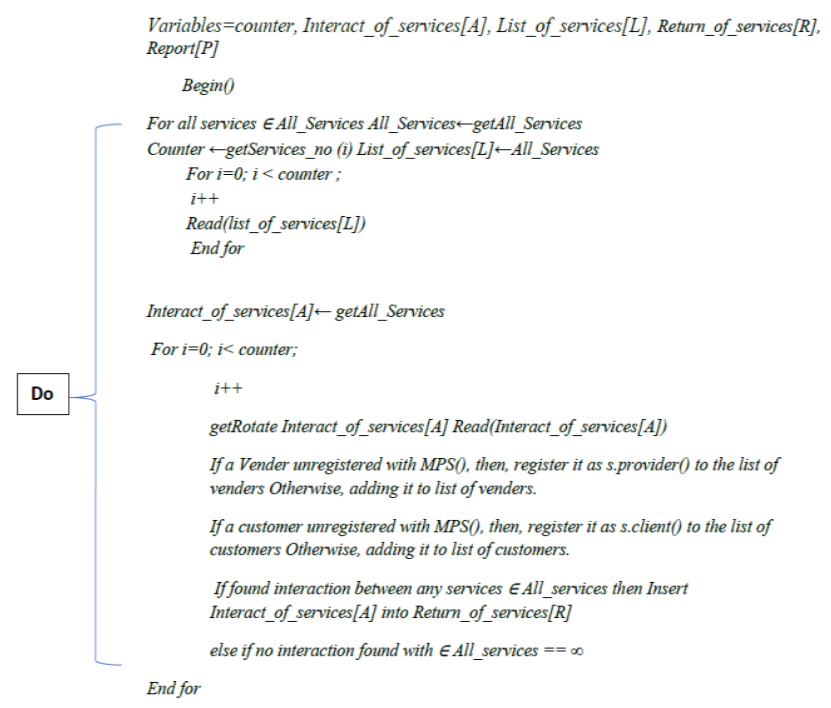

\section{CONCLUSION}

Currently, NFC has become a dynamic service that shows ongoing maturity with the technology revolution. Many telecom industries players, e.g., Google, Samsung, Apple, and Nokia, can embrace and adopt the NFC technology.

We are ongoing to an era of mobile smartphones wallet where cash value that can be stored on a card, phone, or another electronic machine that may be topped up. The phrase wallet has set and used the debit or credit card embedded with a phone instead of carrying pocket money. As a result, this will grant and enable smartphone users worldwide to pay via their handsets mobile phones process for a lake of a wide range of activities i.e.

Published By:

Blue Eyes Intelligence Engineering and Sciences Publication

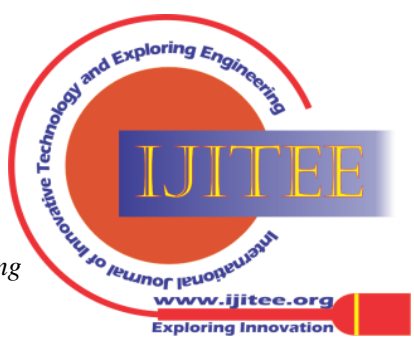


services, digital and hard goods. Still, technology such as Wi-Fi and Bluetooth look like NFC because they give data exchange and wireless communication between digital machines such as smartphones. NFC performs at exploiting a set of electromagnetic radio fields while technologies such as $\mathrm{Wi}-\mathrm{Fi}$ and Bluetooth concentrate on radio transmissions instead. With NFC, having an ability for connecting and launching automatically with each other NFC machine enters the four-inch range. After that, these two machines speak and send prompts to the users. A range of machines using NFC can be either active or passive.

Thus, this paper has developed and demonstrated a technique that permitted to exchange through technology wireless data between the parties within a Web services environment.

Developing such a system is hard work, and it may have many aspects that require exploring the MPS design by granting some features in dynamically changing environments. Therefore, we have implemented the SR barcode program that can work as an NFC technology at many places and then adapt this program to manage the structure of requirements, which will be applying a systematic process that the model criteria can leverage. And then, proving and validating the proposed approach to achieve optimal solution using FSM and JFLAP tools.

Further, the NFC technology has a wide range of investigations that can be done. The NFC technology can be used in several aspects, e.g., Transportation, new smart city, university, and health sector.

\section{REFERENCES}

1. http://www.radio-electronics.com/info/wireless/nfc/nfc-near-field-com munications-technology.php

2. PressReleaseshttp://newsroom.mastercard.com/press-releases/masterpa ss-digital-wallet-launches-in-canada/

3. http://www.interfacemagazine.co.nz/downloads/INTERFACE\%20Less on\%20Plan\%2072\%20-\%20QR\%20Codes.pdf

4. http://www.imc.org/pdi/vcardoverview.html

5. UML Reference Manual, Rumbaugh, Jacobson, and Booch. http://www.engage-consulting.biz/docs/usecaseexample.pdf

6. http://searchsoa.techtarget.com/definition/data-dictionary

7. N J Davies, D Fensel and M Richardson, The future of the Web services, BT Technology Journal • Vol 22 No 1 • January 2004

8. K.Aldriwiesh, Security Policy Architecture for Web Services Environment, PhD dissertation theses , June 19-2012, De Montfort university, Leicester, UK

9. Tanmaya Gupta, ABAP Data Dictionary, ABAP group, Galileo Press, 2011

10. Lawrence M. Muriira, Near Field Communication (NFC) Technology: The Future Mobile Money Service for Kenya, International Journal of Computing and ICT Research, Vol. 6, Issue 1, June 2012

11. Khalid Aldriwish, An Automation Instructor System using Finite State Machine within Web services, in the IJCSNS International Journal of Computer Science and Network Security, VOL.21 No.7, July 2021

\section{AUTHORS PROFILE}

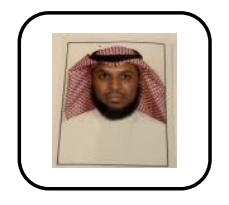

Khalid Aldriwish, received his BSc, MSc, $\mathrm{PhD}$ and ITIL. I am working as assistant professor at Majmaah university since 2013, also I work with a company as IT consultant. I have many publications found in Google scholar, and my research area is security policy, web service security, blockchain.

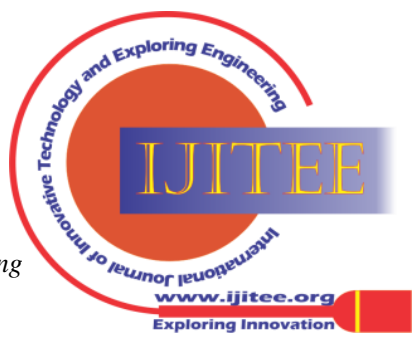

